\title{
Rapid Prototyping Methodology of Lightweight Electronic Drivers for Smart Home Appliances
}

\author{
Trio Adiono, Rachmad Vidya Wicaksana Putra, Maulana Yusuf Fathany, Braham Lawas Lawu, \\ Khilda Afifah, Muhammad Husni Santriaji, Syifaul Fuada \\ Department of Electrical Engineering, School of Electrical Engineering and Informatics, \\ Institut Teknologi Bandung, Indonesia
}

\section{Article Info}

Article history:

Received May 16, 2016

Revised Jul 13, 2016

Accepted Jul 29, 2016

\section{Keyword:}

Home appliances

Lightweight drivers

Rapid prototyping method

Smart home platform

\begin{abstract}
Many researches have been conducted in smart home topic. Mostly, they discussed on the specific aspect of application. On the other side, many applications still can be explored and attached into the system. Several main challenges in designing the application devices are system complexity, reliability, user friendliness, portability, and low power consumption. Thus, design of electronic driver is one of the key elements for overcoming these challenges. Moreover, the drivers have to comply the rules of smart home system, data protocol, and application purpose. Hence, we propose a rapid prototyping methodology on designing lightweight electronic drivers for smart home appliances. This methodology consists of three main aspects, namely smart home system understanding, circuitry concept, and programming concept. By using this method, functional and lightweight drivers can be achieved quickly without major changes and modifications in home electrical system. They can be remotely controlled and monitored anytime and from anywhere. For prototyping, we design several drivers to represent common electronic and mechanical based applications. Experimental results prove that the proposed design methodology can achieve the research target.
\end{abstract}

Copyright (C) 2016 Institute of Advanced Engineering and Science. All rights reserved.

\section{Corresponding Author:}

Trio Adiono,

Department of Electrical Engineering,

School of Electrical Engineering and Informatics, Institut Teknologi Bandung,

Jl. Ganesha No. 10, Bandung 40132, Indonesia.

Email: tadiono@stei.itb.ac.id

\section{INTRODUCTION}

Smart Home concept has become one of technology trends in last few years. It grows rapidly and gain massive popularity because of the demands [1]. Many experimental projects were conducted by universities in this field, such as University of Colorado with The Adaptive House, Sweden Interactive Institute with comHOME, MIT with House_n, and Georgia Institute of Technology with The Aware Home [2]. Separately, many companies are also working in this area with their own projects (e.g. Control4, Crestron, AMX, etc).

Based on literatures, researches on smart home design could be generally divided into four main categories, namely: (1) platform design, (2) authentication and security design, (3) Graphical User Interface (GUI) design, and (4) end-device driver design. Platform design covers communication scheme [3], network topology [4], energy management [5]-[8], controlling scheme [9]-[10], and sensory schemes (e.g. anti-theft [11], fire detection [12], toxic gases detection [13]-[14]). Authentication and security design covers all security issues (e.g. cryptography, biometric key, personal identification number, or specific algorithm [15][18]). GUI design covers all user interface issues (e.g. web-based [19], smartphone-based [20]); and (4) driver design covers electronic circuitry and its programming. 
Actually, many application devices still can be explored and developed for applications, because almost every single home appliance could be modified in order to be connected into smart home system. From [21] and [22], we can see that there are two major categories of applications: electronic and mechanical based devices. Main challenges in designing those application devices are about system complexity, reliability, user friendliness, portability, and low power consumption. Thus, design of electronic driver is one of the key elements for overcoming the challenges. Moreover, rules of smart home system, data protocol, and application purpose also have to be followed in term of design implementation.

In this research, we propose a general and rapid prototyping methodology for designing lightweight electronic drivers for any smart home appliances. By using this technique, several main functions can be achieved quickly without major changes and modifications in home electrical system. For prototyping purposes, we design electronic drivers to represent and support both electronic and mechanical targets, such as: humidity-temperature sensors, general power socket, ambient colored lamp, door lock, fan speed control, and curtain control. All of them can be remotely controlled and monitored using mobile application (e.g. smart-phone apps), anytime and from anywhere. Experimental results prove that the proposed method achieve the research targets (i.e. low system complexity, high reliability, user friendly, high portability, and low power).

This paper is organized into a number of sections. The first section is introduction and explanation on the research background, problems, and solution. The second one explains the proposed design methodology. It explores main idea of the design and its related discussions. Following in the third and fourth sections are prototypes and evaluations. They describe the design samples on several functions of appliances and their evaluation results. Last three sections are conclusions, acknowledgment, and references.

\section{PROPOSED DESIGN METHODOLOGY}

\subsection{Smart Home System}

In order to design the end-devices, firstly we need to determine how data protocol dan smart home system work. Here, we decide to use proposed data protocol and smart home system architecture in [23]-[25], because of the simplicity and compatibility. Data protocol provides 3-byte for header, 1-byte for address, 1byte for packet initialization, n-byte for payload and 1-byte for checksum, as presented in Table 1 . Meanwhile, illustration of the smart home system is shown in Figure 1. The system has two environments, indoor and outdoor. Indoor section uses Wireless Sensor Network (WSN) configured in mesh topology, while outdoor section uses mobile data communication scheme. These two environments are connected by gateway. Using this design approach, home appliances can be controlled and monitored, anytime and from anywhere.

WSN is a new generation of network which its main purpose is to collect data from the surrounding environment of networked sensors [26]. It is formed by small and low-cost nodes which have limitations in energy, memory, and processing capacity [27]. In WSN communication scheme, three communiaction protocols can be used (i.e. ZigBee, Bluetooth, WiFi). ZigBee protocol has simple data structure and low data rate, thus it consumes low power. Hence, it is suitable as the backbone communication of WSN. Bluetooth protocol has medium data rate and compatibility to connect to smart phone. Hence, it is used as a communication scheme between user applications to the WSN system. Meanwhile, WiFi has the highest data rate among these three protocols and compatibility to connect to smart phone. Hence, WiFi is used for intensive applications, such as video streaming.

Table 1. Data protocol format

\begin{tabular}{ccccc}
\hline Header & Address & Packet Init & Data Payload & Checksum \\
\hline 3-byte & 2-byte & 1-byte & n-byte & 1-byte \\
\hline
\end{tabular}




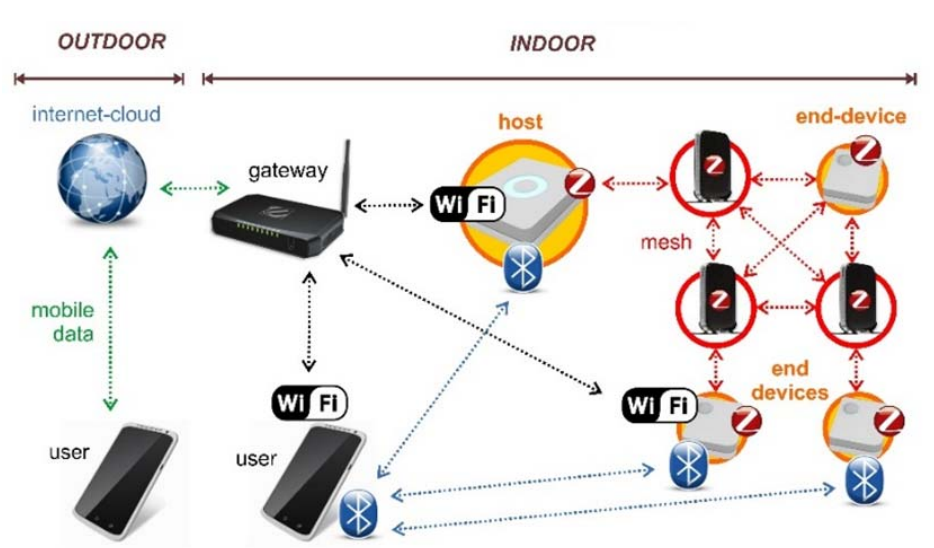

Figure 1. Smart home system architecture

\subsection{Circuitry Concept}

Circuitry concept is one of the important aspects that affect the whole design complexity of electronic driver. Proposed electronic circuit concept is shown in Figure 2. There are six main modules involved: power supply, antenna, communication module, microcontroller, specific electronic circuit, and indicator. Power supply gives energy to whole circuit (i.e. communication module, microcontroller, and specific circuit). These modules will be supported by indicator, since indicator module is the one that gives us information status about the driver. Thus, indicator will be affected by communication module, microcontroller, and specific circuit. In this research, communication module uses ZigBee and microcontroller uses STM32. Meanwhile, specific electronic circuit could be various since it depends on the application target. By using this simple circuitry, hopefully low-complexity hardware circuit can be designed in a small dimension of Printed Circuit Board (PCB) and its designated packaging. For power supply, there are two possible scenarios, direct supply from AC power outlet or battery. If the designated applications need a high mobility and portability, battery is a better choice. Thus, rechargeable battery design and its level indicator are considered. Meanwhile, if the applications need power outlet as main source, battery may be neglected to consider design simplicity.

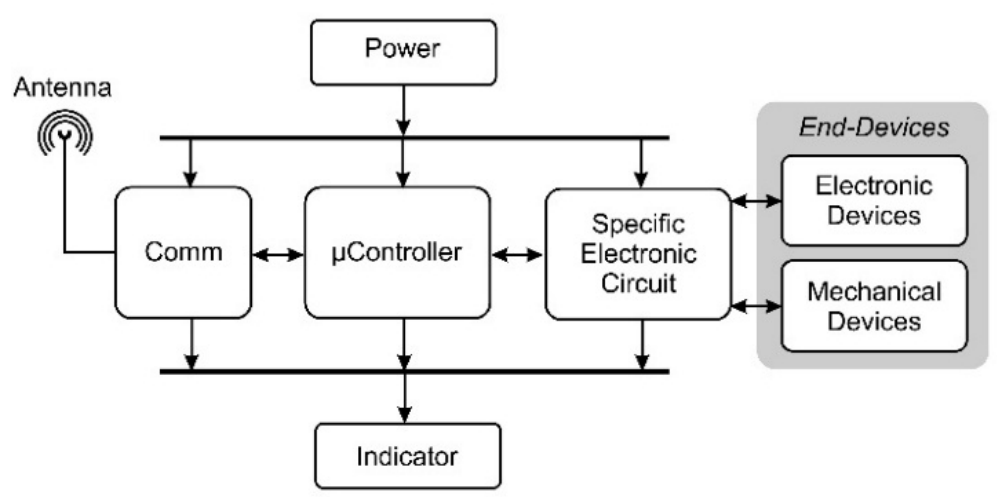

Figure 2. Basic electronic circuit schematic

\subsection{Programming Concept}

Microcontroller programming is another crucial factor in determining how the driver will respond to the specific event. In order to make the driver works efficiently, such lightweight program is needed. Illustration of the proposed programming scheme is presented in Figure 3. In the setup section, we need to declare variable and library, create interrupt handler, and create specific functions. In the main section, routine program is the center of programming. It will be supported by library, configuration, specific functions, and interrupt handler. One of the tricky things here is to use the right timer for programming. Wrong decision in using source of timer can cause errors or invalid outcomes. Creating such programming 
block and its behavior first before doing actual programming is recommended, since these steps could help to map and thoroughly design the program. By using this design block, we can also thoroughly troubleshoot the program if errors or invalid outcomes occur in the testing and evaluation phase.

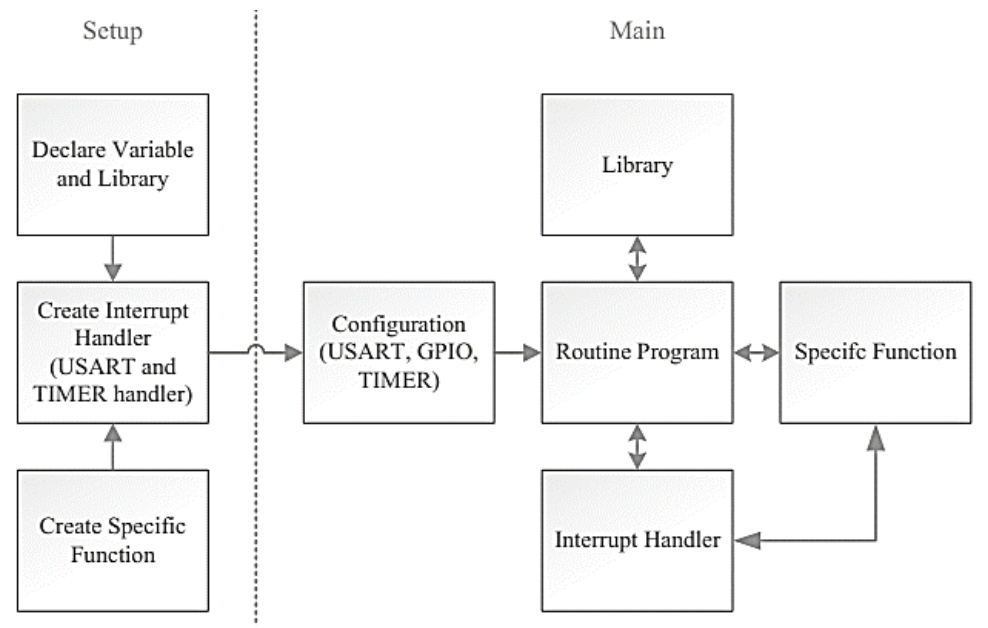

Figure 3. Proposed programming structure

\section{DRIVER PROTOTYPES FOR HOME APPLIANCES}

\subsection{Door Lock Driver}

The main idea of the door lock driver is to provide an electronic circuit which can pull and release the solenoid, in order to represent locking and unlocking condition for the door. Illustration of the circuit is given in Figure 4. A digital pin of microcontroller gives bias signal to transistor and the solenoid will give a response with pulling or relaxing itself. A normally-closed type of solenoid is used, since it is better in saving power consumption. The solenoid will pull itself only when the bias is active. This active condition is expected to be short enough, thus the power usage can be minimized. Function of diode IN4007 is protecting the other components when the solenoid changes its position quickly. We can see that the circuit complies with the proposed concept and it only needs 3 basic components (i.e. transistor, resistor, and diode) for controlling the solenoid.

\subsection{Curtain Control Driver}

The main idea of the curtain control driver is to provide an electronic circuit which can control the movement of curtain. Moreover, we can control how far the curtain should open. Here, we use stepper motor as mechanical target device, because we can set how far the stepper motor should roll. Illustration of the circuit is given in Figure 5. Four digital pins of microcontroller give Pulse Width Modulation (PWM) signal to motor driver. Motor driver ULN2003A will translate the received command into control signal for stepper motor. The circuit complies with the proposed concept and only needs single motor driver, 4 Light Emitting Diodes (LEDs), and 4 resistors for controlling the stepper motor.

\subsection{Fan Control Driver}

The main idea of the fan control driver is to provide an electronic circuit which can dynamically change the speed of fan. For prototyping purpose, we use Direct Current (DC) motor as mechanical target device. Illustration of the circuit is given in Figure 6. A digital pin of microcontroller gives bias signal to transistor and DC motor will respond to start rotating or stop rotating. The circuit is the same concept with door lock driver, but the differences are in the programming and biasing condition. In order to achieve its dynamic bias, microcontroller gives PWM signal which its pulse width determines the rotational speed of DC motor. Function of diode IN4007 is protecting the other components when the solenoid changes its position quickly. The circuit complies with the proposed concept and only needs 3 basic components (i.e. transistor, resistor, and diode) for controlling the DC motor. 


\subsection{General Power Socket Driver}

The main idea of this general power socket driver is to provide an electronic circuit which can switch ON/OFF as instructed. Relay is used to perform ON/OFF switching function. The circuit needs to control when the relay is connected to the power source $(\mathrm{ON})$ or the ground (OFF). Figure 7 shows the circuit schematic. Function of diode IN4007 is protecting the components when the relay changes its switch position quickly. Because, when relay switchs its position quickly, high voltage will be switched in short time. Moreover, in order to keep the circuit stable, optocoupler is used to isolate microcontroller signal from the noise of high voltage. The circuit complies with the proposed concept and only needs 4 basic components (i.e. transistor, resistor, diode, optocoupler) for controlling the relay.

\subsection{Humidity and Temperature Sensor Driver}

The main idea of this humidity-temperature (H-T) sensor driver is to support the sensor functionalities. In this research, we use sensor DHT11. Figure 8 shows the circuit. It is very simple, since it only needs a resistor. Choosing the accurate value of resistor will improve the accuracy of the sensor. We can adjust here by using potensiometer in order to get the best value of resistance in delivering valid data of humidity and temperature. This circuitry is one of the simplest hardware in the project.

\subsection{Ambient Colored Lamp Driver}

The main idea of this ambient colored lamp driver is to provide an electronic circuit which can control the colors illumination and brightness of Light Emitting Diode (LED) lamp. Color of the lamp can be red $(R)$, green $(G)$, blue $(B)$, or their combination (RGB). In order to design a proportional driver, we need to understand the characteristic the RGB lamp itself. Thus, we can use the appropriate components for driving the each LED color. Figure 9 shows the circuit schematic. It applies to each red, green and blue colors, but different values of resistors could apply. The $R_{1}-R_{11}$ are resistors which help to create appropriate voltage to the LEDs, meanwhile the $R_{12}$ is used for biasing of the transistor.

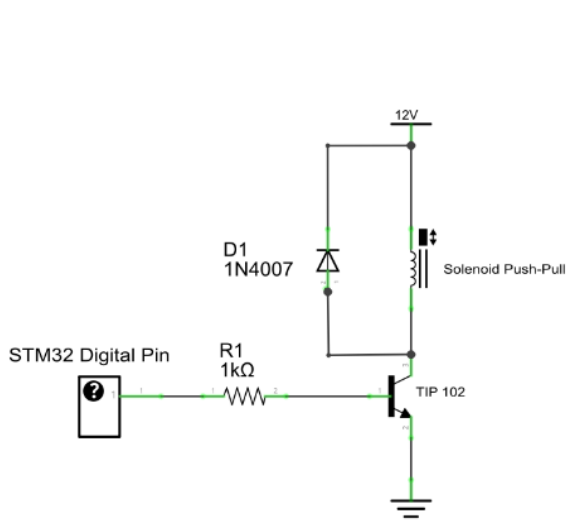

Figure 4. Specific circuit for door lock

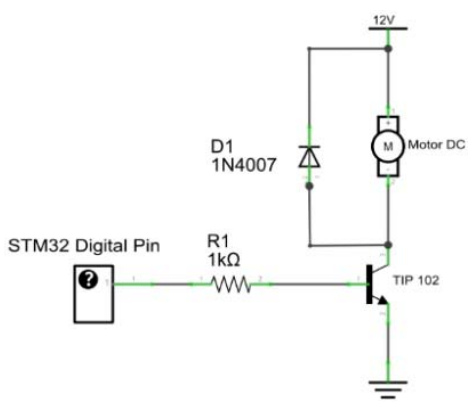

Figure 6. Specific circuit for fan

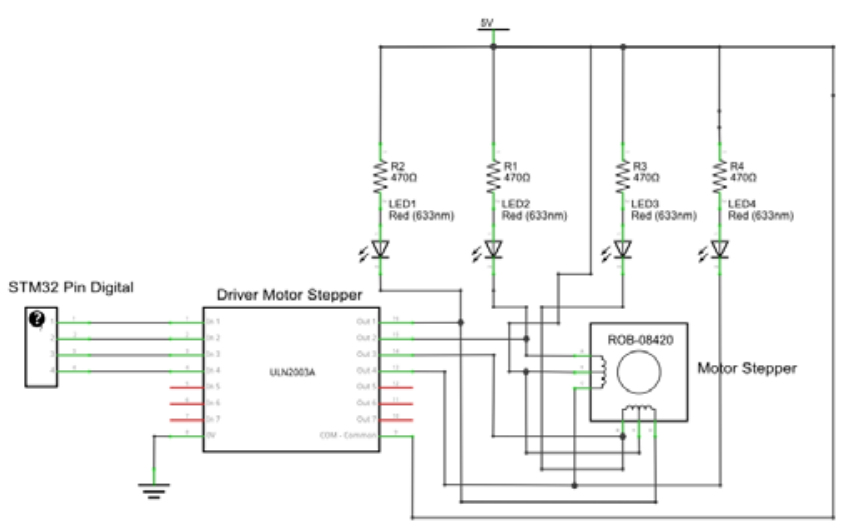

Figure 5. Specific circuit for curtain control

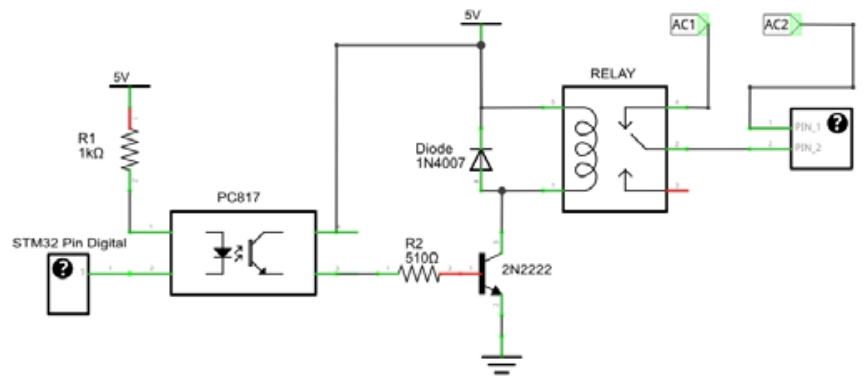

Figure 7. Specific circuit for power socket driver 


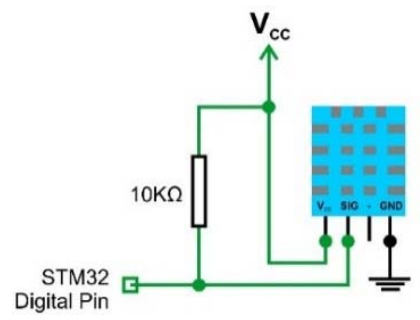

Figure 8. Specific circuit for H-T sensor

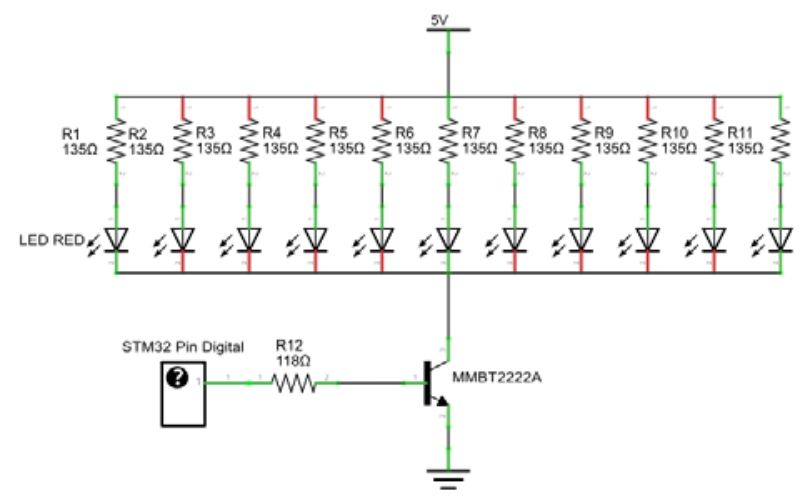

Figure 9. Specific circuit for colored RGB lamp

\section{EVALUATIONS}

\subsection{Functionalities}

In the functional evaluations, each prototype is examined. For door lock driver, we examine that if the "pull" command is given, solenoid responds shortly to pull itself. It represents open condition. When reverse command "relaxed" is given, solenoid is relaxed and back to lock condition. Figure 10 is the snapshot from the test. For curtain control driver, we examine the response of stepper motor. Stepper motor is controlled to roll as far as desired on a particular direction (i.e. clockwise or counter-clockwise). Figure 11 is the snapshot from the test. Curtain can open and close as expected. For fan control driver, we examine the response of DC motor on the different speed. Results show that DC motor can keep up with the given commands on different speed. The driver of DC motor is working properly as shown in Figure 12.

For general power socket driver evaluation, we use several appropriate appliances to connect (i.e. lamp, LCD monitor, television, fan, etc). It evaluates that the relay is working properly and the connected appliances respond ON/OFF accordingly. It is shown in Figure 13. For ambient colored RGB lamp driver, combinations of colors are working well as shown in Figure 14. Lastly, for H-T sensor driver evaluations, results are given in Figure 15. Result of sensing is shown in Android-based mobile application in smartphone. It indicates that the driver is working properly, giving the valid data of humidity and temperature.

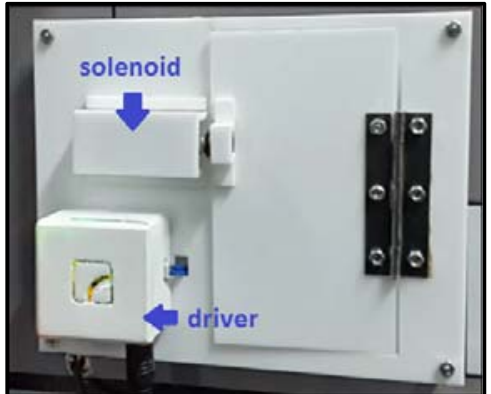

(a)

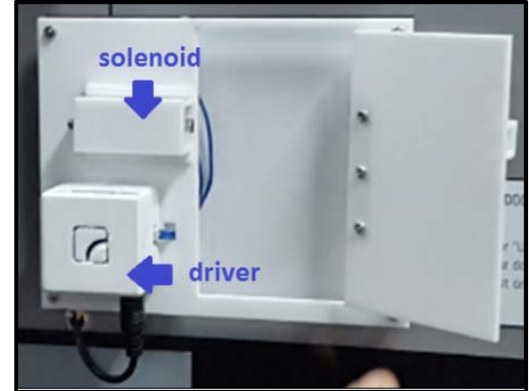

(b)

Figure 10. Functional evaluation on door lock driver: (a) locked, (b) opened 


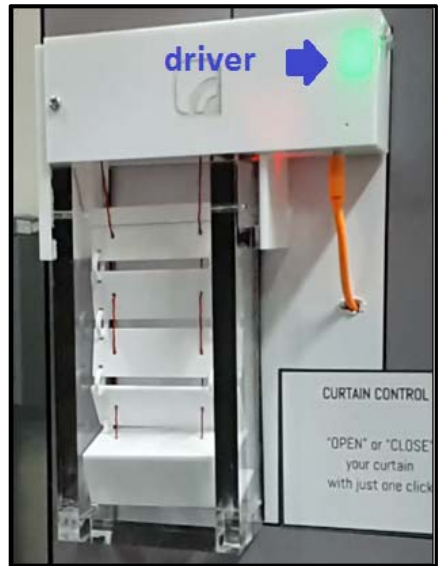

(a)

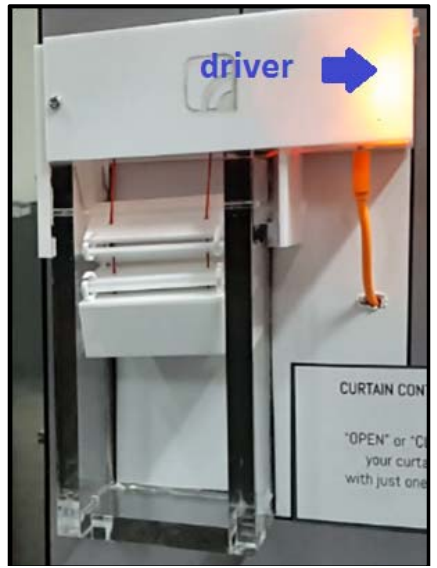

(b)

Figure 11. Functional evaluation for curtain driver: (a) closed, (b) opened

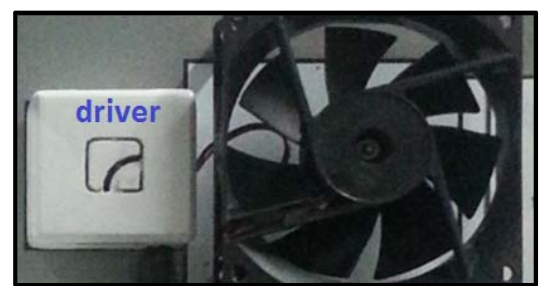

(a)

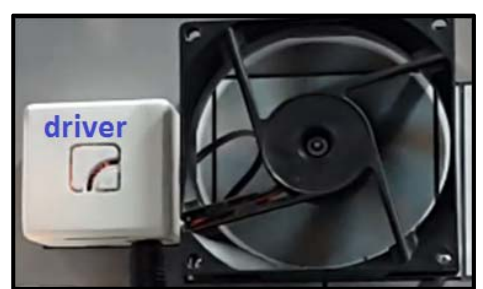

(b)

Figure 12. Functional evaluation for fan driver: (a) OFF, (b) ON

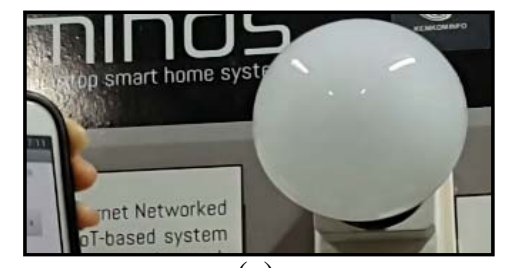

(a)

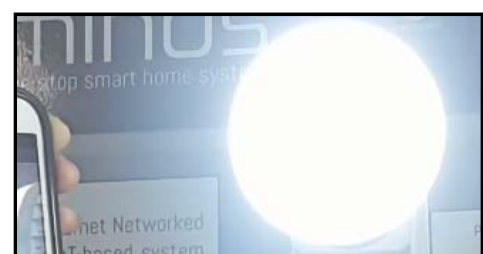

(b)

Figure 13. Functional evaluation for power socket driver: (a) OFF, (b) ON

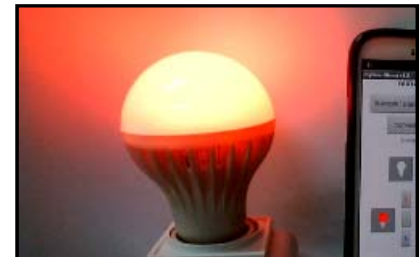

(a)

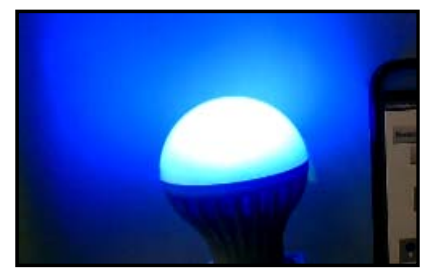

(c)

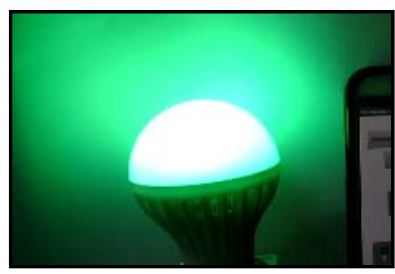

(b)

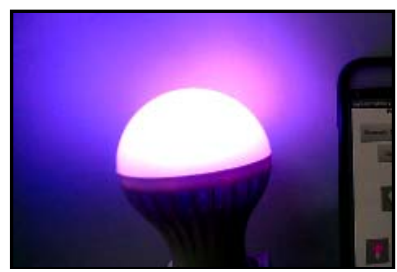

(d) 


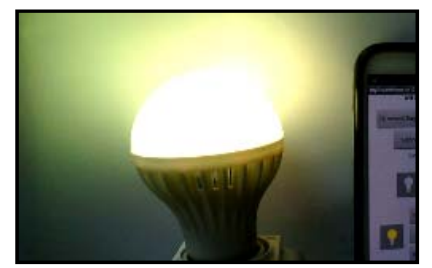

(e)

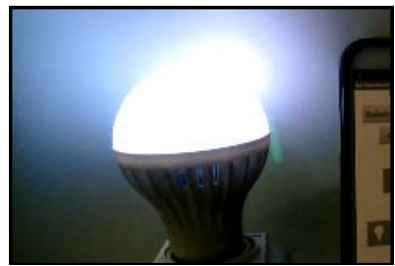

(f)

Figure 14. Functional evaluation for ambient lamp driver: (a) red, (b) green, (c) blue, (d) magenta, (e) yellow, (f) white

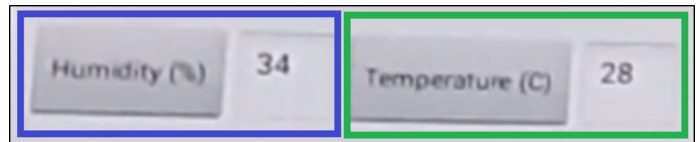

Figure 15. Functional evaluation for $\mathrm{H}-\mathrm{T}$ sensor driver

\subsection{Power Consumption}

Power consumption is evaluated for each device. Results of measurement are presented in Table 2. We can see that the lowest current is used when idle condition. Meanwhile, in the operating condition, current level rises. The highest increasing is on RGB lamp application. It is acceptable because RGB lamp needs a stable current for illumination. The second highest is door lock application. It is predicted because this application needs enough current to retain the position of the solenoid when activated. The third until the last are curtain control, fan control, power socket, and $\mathrm{H}-\mathrm{T}$ sensor respectively. From this measurements, we can see that the most power hungry device is door lock, with $\sim 7$ Watt. But, the best part is that we only use the door lock in short of time when opening the door. Moreover, the most frequently used device is H-T sensor and it only consumes $246 \mathrm{~mW}$ when operating. From these data, we can see that low power consumption is able to be achieved using the proposed methodology.

Table 2. Power consumption on devices

\begin{tabular}{cccrrr}
\hline \multirow{2}{*}{ Device } & \multirow{2}{*}{ Voltage $(\mathrm{V})$} & \multicolumn{2}{c}{ Current $(\mathrm{mA})$} & \multicolumn{2}{c}{ Power (mW) } \\
& & Idle & Operating & Idle & Operating \\
\hline H-T Sensor & 5 & 42.6 & 49.2 & 213.0 & 246.0 \\
Power Socket & 5 & 49.9 & 123.7 & 249.5 & 618.5 \\
Door Lock & 12 & 42.3 & 587.5 & 507.6 & 7050.0 \\
Fan Control & 12 & 43.1 & 145.1 & 517.2 & 1741.2 \\
Curtain Control & 5 & 42.1 & 317.2 & 210.5 & 1586.0 \\
RGB Lamp & 5 & 42.8 & 603.1 & 214.0 & 3015.5 \\
\hline
\end{tabular}

\section{CONCLUSIONS}

In this paper, rapid prototyping methodology of lightweight electronic drivers for smart home appliances is proposed. Since many desired applications can be explored and attached into the smart home system, such methodology is needed to design the effective and efficient electronic drivers and keep the system complexity being low and lightweight. The proposed method can overcome several main challenges like low system complexity, high reliability, user friendly, high portability, and low power consumption. In prototyping evaluations, several drivers with representaive functions can be achieved and covered quickly without major changes and modifications in home electrical system. They perform and work properly as expected. It shows that the proposed methodology is synchronized with the research target and expectation.

\section{ACKNOWLEDGEMENTS}

This research is supported by “Program Penelitian Unggulan Perguruan Tinggi DIKTI 2016” from The Ministry of Research and Technology for Higher Education (RISTEK-DIKTI), Republic of Indonesia. 


\section{REFERENCES}

[1] M. Chan, et al., "A review of smart homes: present state and future challenges," Computer Methods and Programs in Biomedicine, vol/issue: 91(1), pp. 55-81, 2008.

[2] F. K. Aldrich, "Smart homes: past, present and future,” Inside Smart Home, Chapter II, Spinger, London.

[3] A. C. Olteanu, et al., "Enabling mobile devices for home automation using ZigBee,” Proc. of Int. Conf. on Control Systems and Computer Science, pp. 189-195, 2013.

[4] Z. Song, "Zigbee network tree routing algorithm based on energy balance," Int. J. of Smart Home, vol/issue: 9(4), pp. 85-96, 2015.

[5] J. Lu, et al., "The smart thermostat: using occupancy sensors to save energy in homes," Proc. of the 8th ACM Conf. on Embedded Networked Sensor Systems, pp. 211-224, 2010.

[6] A. Fensel, et al., "SESAME-S: semantic smart home system for energy effciency," Informatik Spektrum, vol/issue: 36(1), pp. 46-57, 2013.

[7] P. K. Choubey, et al., "Power efficient, bandwidth optimized and fault tolerant sensor management for IoT in smart home,” Proc. of Int. Advance Computing Conf., pp. 366-370, 2015.

[8] K. Nair, et al., "Optimizing power consumption in IoT based wireless sensor networks using bluetooth low energy,” Proc. of Int. Conf. on Green Computing and Internet of Things, pp. 589-593, 2015.

[9] J. Li, et al., "A novel remote monitoring and control system based on GSM for home security," Int. J. of Online Engineering, vol/issue: 11(4), pp. 34-38, 2015.

[10] Y. Liang and S. Wan, "The design of smart home control system," Proc. of Int. Conf. on Instrumentation and Measurement Computer Communication and Control, pp. 311-314, 2014.

[11] D. Erwanto, "Penerapan sistem pengaman rumah menggunakan sensor passive infrared receiver," J. Cendekia, vol/issue: 11(1), pp. 16-21, 2013.

[12] H. S. Kong, et al., "Development of smart phone applications linked with fire alarm control panel in automatic fire detection system,” Int. J. of Smart Home, vol/iisue: 10(2), pp. 11-18, 2016.

[13] L. Guo, et al., "Design for indoor environment monitoring system based on embedded system and multi-sensor data fusion algorithm,” Int. J. of Smart Home, vol/issue: 10(1), pp. 31-40, 2016.

[14] Y. Zhang and Y. Zhao, "Design \& implementation of an air quality monitoring system for indoor environment based on microcontroller,” Int. J. of Smart Home, vol/issue: 9(11), pp. 301-312, 2015.

[15] R. V. Rodríguez, et al., "Footstep recognition for a smart home environment," Int. J. of Smart Home, vol/issue: 2(2), pp. 95-110, 2008.

[16] J. Jeong, et al., "Integrated OTP-based user authentication scheme using smart cards in home networks," Proc. of the 41st Hawaii Int. Conf. on System Sciences, pp. 1-7, 2008.

[17] K. Y. Lian, et al., "Home safety handwriting pattern recognition system," Proc. of Int. Conf. on Cognitive Informatics and Cognitive Computing, pp. 477-483, 2012.

[18] M. H. Assaf, et al., "Sensor based home automation and security system," Proc. of Int. Instrumentation and Measurement Technology Conf., pp. 722-727, 2012.

[19] F. Arifiyanto, et al., "Perancangan prototype web-based online smart home controlled by smartphone," $J$. Transient, vol/issue: 2(4), pp. 916-923, 2013.

[20] A. Giyartono and P. E. Kresnha, "Aplikasi Android pengendali lampu rumah berbasis mikrokontroler ATmega328,” Seminar Nasional Sains dan Teknologi, pp. 1-9, 2015.

[21] B. L. Lawu, et al., "Prototyping design of mechanical based end-devices for smart home applications," Proc. of 2016 4th Int. Conf. on Information and Communication Technology (ICoICT), pp. 297-302, 2016.

[22] T. Adiono, et al., "Prototyping design of electronic end-devices for smart home applications," Proc. of 2016 The IEEE Region 10 Symposium (TENSYMP), pp. 1-5, 2016.

[23] M. Y. Fathany and T. Adiono, "Wireless protocol design for smart home on Mesh Wireless Sensor Network," Proc. of Int. Symposium on Intelligent Signal Processing and Communication Systems (ISPACS), pp. 462-267, 2015.

[24] T. Adiono, et al., "Smart home platform based on optimized wireless sensor network protocol and scalable architecture," Proc. of Int. Conf. on Telecommunication Systems Services and Applications (TSSA), pp. 1-5, 2015.

[25] T. Adiono, et al., "Desain sistem rumah cerdas berbasis topologi mesh dan protokol wireless sensor network yang efisien,” Jurnal Informatika, Sistem Kendali dan Komputer (INKOM), vol/issue: 9(2), pp. 65-72, 2015.

[26] M. Jafari and H. Khotanlou, "A Routing Algorithm Based on Ant Colony, Local Search and Fuzzy Inference to Improve Energy Consumption in Wireless Sensor Networks,” Int. J. of Electrical and Computer Engineering (IJECE), vol/issue: 3(5), pp. 640-650, 2012.

[27] J. Rezazadeh, et al., "Fundamental Metrics for Wireless Sensor Networks localization,” Int. J. of Electrical and Computer Engineering (IJECE), vol/issue: 2(4), pp. 452-455, 2012. 


\section{BIOGRAPHIES OF AUTHORS}

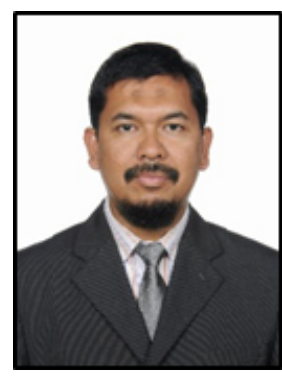

Trio Adiono received B.Eng on Electrical Engineering and M.Eng on Microelectronics from Institut Teknologi Bandung (ITB), Indonesia, in 1994 and 1996. He obtained his Ph.D. degree in VLSI Design from Tokyo Institute of Technology, Japan, in 2002. He received the "Second Japan Intellectual Property Award" in 2000 from Nikkei BP for his research on "Low Bit-rate Video Communication LSI Design". He also holds a Japanese Patent on "High Quality Video Compression System". Currently, he is a lecturer at the School of Electrical Engineering and Informatics, a Head of the Microelectronics Center and IC Design Laboratory, ITB. He currently serves as a chair of the IEEE SSCS Indonesia Chapter. His research interests include VLSI, Signal and Image Processing, Smart Card, Electronics Solution Design and Integration.

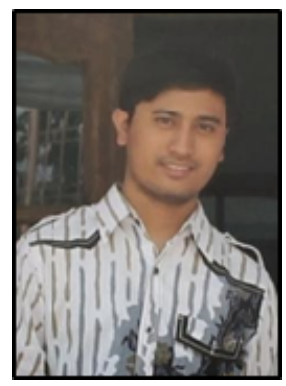

Rachmad Vidya Wicaksana Putra received B.Sc on Electrical Engineering in 2012 and M.Sc on Microelectronics with a distinction Cum Laude in 2015, both from Institut Teknologi Bandung (ITB). He received an Indonesia Endowment Fund for Education (IEFE/LPDP) Scholarship for his master study and potentially for his $\mathrm{PhD}$ study. He was involved in several projects (i.e. Heart Rate Sensing, Military Digital Radar Display, and SDR). Currently, he is managing several researches in Microelectronics Center ITB (i.e. RF Monitor, Smart Home, etc). He is also preparing to start PhD study at KTH Sweden this year. His interests are about VLSI, SoC/NoC, Neuromorphic, Signal Processing, IoT and Visible Light Communication.

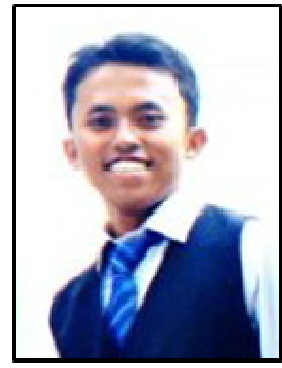

Maulana Yusuf Fathany received his B.A.Sc on Computer Engineering from Politeknik Elektronika Negeri Surabaya, Institut Teknologi Sepuluh Nopember (ITS), Indonesia in 2013. He received M.Sc on Microelectronics major from Institut Teknologi Bandung (ITB), Indonesia in 2016. His research interests are Software Engineering, Embedded Systems, Wireless Sensor Network (WSN), and Internet of Things. His life motto is "Life without Fear, Die without Regret"

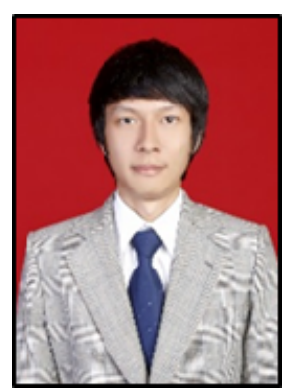

Braham Lawas Lawu received B.Eng on Electrical Engineering from Maranatha Christian University in 2014. He is currently Master Student in Microelectronics Major at Institut Teknologi Bandung (ITB), Indonesia. His research interests are mainly about Analog Instrumentation and Measurement, Analog IC Design, Control, Automation, RF, Power Electronics, Embedded Systems, and Internet of Things for Smart Home.

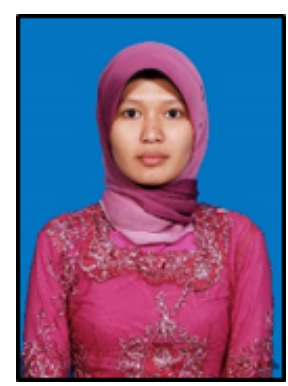

Khilda Afifah received the B.Ed on Electrical Engineering Education, Universitas Pendidikan Indonesia in 2014. She is currently Master Student on Microelectronics Program in School of Electrical Engineering and Informatics, Institut Teknologi Bandung (ITB), Indonesia. Her research interests are analog front end design for Smart Card, CMOS technology, and Internet of Things for Smart Home. 


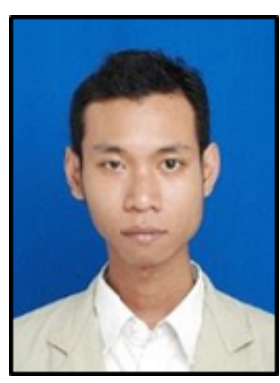

Muhammad Husni Santriaji received the B.Sc on Electronics and Instrumentation from Universitas Gadjah Mada (UGM), Indonesia in 2011. He received M.Sc on Microelectronics from Institut Teknologi Bandung (ITB), Indonesia in 2016. He is also preparing to start his PhD study at University of Chicago, United States this year. He has been involved in several researches; one of them was in collaboration under research-scholarship program from University of Chicago. His research interests are reconfigurable computing, VLSI, and Internet of Things for Smart Home.

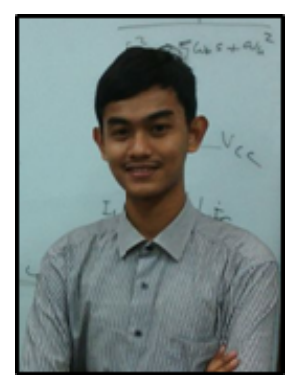

Syifaul Fuada received B.Ed degree on Electrical Engineering Education major from State University of Malang (UM), Indonesia in 2014. Now, currently he is pursuing his M.Sc degree in Institut Teknologi Bandung (ITB), Indonesia on Microelectronics. He received Panasonic Scholarship for his master study. He holds two pending patents and several achievements such as, the best poster in SPRINT 2014, the best poster in KIPNAS XI in 2015 and one of the 106 Indonesian Innovators by BIC-RISTEK DIKTI in 2014. 